\title{
Effects of Preventive Long-Term Treatment with Strontium Ranelate and Zoledronic Acid on Bone Quality in Ovariectomized Rats
}

\author{
Marta Martín-Fernández ${ }^{1}$, Marina Gómez-Chinchón ${ }^{1}$, Luis Álvarez ${ }^{2}$, Blanca Torrubia ${ }^{1}$, \\ Manuel Díaz-Curiel ${ }^{3}$, David Guede ${ }^{4}$, Jose Ramón Caeiro ${ }^{5}$, Cristobalina Rodríguez-Álvarez ${ }^{6}$, \\ Concepción de la Piedra ${ }^{1}$ \\ ${ }^{1}$ Biochemistry Investigation, Sanitary Research Institute Jimenez Diaz Foundation, Madrid, Spain \\ ${ }^{2}$ Spinal Pathology Unit, Sanitary Research Institute Jimenez Diaz Foundation, Madrid, Spain \\ ${ }^{3}$ Internal Medicine, Sanitary Research Institute Jimenez Diaz Foundation, Madrid, Spain \\ ${ }^{4}$ Trabeculae, Limited Society, Technological Park of Galicia, Ourense, Spain \\ ${ }^{5}$ Traumatology and Orthopedic Surgery, Universitary Hospital of Santiago de Compostela, Santiago de Compostela, Spain \\ ${ }^{6}$ Area of Preventive Medicine and Public Health, Health Sciences Faculty, Medicine, University of La Laguna, Tenerife, Spain
}

\section{Email address:}

martamartinfernandez@gmail.com (M. Martín-Fernández), mgomezc@fjd.es (M. Gómez-Chinchón), lalvarez@fjd.es (L. Álvarez), blanca.torrubia@quironsalud.es (B. Torrubia),mdcuriel@fjd.es (M. Díaz-Curiel), guede.d@icloud.com (D. Guede), jrcaeiro@telefonica.com (J. R. Caeiro), crrodrig@ull.es (C. Rodríguez-Álvarez), cpiedra@fjd.es (C. de la Piedra)

\section{To cite this article:}

Marta Martín-Fernández, Marina Gómez-Chinchón, Luis Álvarez, Blanca Torrubia, Manuel Díaz-Curiel, David Guede, Jose Ramón Caeiro, Cristobalina Rodríguez-Álvarez, Concepción de la Piedra. Effects of Preventive Long-Term Treatment with Strontium Ranelate and Zoledronic Acid on Bone Quality in Ovariectomized Rats. American Journal of Clinical and Experimental Medicine.

Vol. 4, No. 6, 2016, pp. 191-200. doi: 10.11648/j.ajcem.20160406.16

Received: September 3, 2016; Accepted: September 18, 2016; Published: November 15, 2016

\begin{abstract}
The aim of this work was to study the effects on bone quality produced by long-range treatment with zoledronic acid (ZA) or strontium ranelate (SrR) in ovariectomized rats. Sixty 6-month-old female Wistar rats were divided: SHAM (n=15), simulated intervention; OVX $(\mathrm{n}=15)$, ovariectomized; OVX+ZA $(\mathrm{n}=15)$, ovariectomized treated with ZA $(0.083 \mathrm{mg} / \mathrm{kg}$ i. $\mathrm{v}$. at the beginning of the study); and $\mathrm{OVX}+\mathrm{SrR}(\mathrm{n}=15)$, ovariectomized treated with $\mathrm{SrR}(0.033 \mathrm{~g} / \mathrm{kg} /$ day by oral gavage). Rats were sacrificed 8 months later. Femoral (F) and lumbar (L) bone mineral density (BMD), trabecular and cortical microstructure, biomechanical testing and Raman spectrometry were performed. FBMD and LBMD decreased in OVX rats with no changes with SrR. ZA treatment prevented changes to BMD. Ovariectomy produced a reduction in micro-CT parameters, while SrR treatment did not avoid these changes. ZA treatment increased micro-CT parameters with respect to the SHAM group. The microstructural parameters of the cortical region were not modified in any of the groups. Ovariectomy produced a decrease in biomechanical parameters that was maintained with SrR. ZA treatment produced an increase in these parameters being higher than those of the SHAM group. Ovariectomy and treatments did not produce differences in crystallinity or substitution index. Mineralization index (MI) decreased in the OVX group. SrR did not revert this effect though ZA avoided it. According to our results preventive treatment with SrR did not revert the alterations in bone quality due to ovariectomy in rats. Treatment with ZA not only reverted the effects of ovariectomy, but also improved bone quality with respect to control rats.
\end{abstract}

Keywords: Ovariectomized Rats, Zoledronic Acid, Strontium Ranelate

\section{Introduction}

Due to the prevalence and severe consequences of postmenopausal osteoporosis, many pharmacological treatments have been developed to prevent or treat the disease. The drugs developed for these purposes can be classified into 3 main groups: bone resorption inhibitors, formation stimulators, and those with dual effects, which 
inhibit bone resorption and stimulate bone formation.

Within the group of agents that inhibit bone resorption, oral bisphosphonates have gained consideration over recent years as a first-line treatment option for osteoporosis, binding to bone and inhibiting osteoclast function. Of these, etidronate, alendronate, risedronate, and ibandronate are employed most frequently.

Poor adherence is a well-known problem with osteoporosis treatment [1]. For this reason, administration of bisphosphonates has been changed to weekly or monthly dosing, and ibandronate is also available as an intravenous injection every 3 months. Zoledronic acid (ZA) is the only bisphosphonate that has been developed exclusively for intravenous use, featuring an attractive regimen of annual infusions for the treatment of osteoporosis, thereby ensuring therapeutic adherence [2].

Strontium ranelate has a dual mechanism of action, activating bone formation and decreasing bone resorption [3].

The above-mentioned treatments produce a significant decrease in fracture risk. However, more studies addressing the effects of these therapies on bone quality are required [4]. Bone quality is the result of the association between bone mass amount (BMD), microstructural parameters e.g., trabecular number, separation, thickness, cortical structure, trabecular pattern factor, degree of anisotropy and mineralization, among others. Bone remodeling is also responsible for mineralization degree depending of a high or low activity. Bisphosphonates produce a significant decrease in bone remodeling, and strontium ranelate increases bone formation and decreases bone resorption. These different actions on bone remodeling may affect bone structure in different ways. The end point would be different extrinsic and intrinsic biomechanical properties. Previous works evaluated which structural parameters (other than bone mass) are the best predictors for changes in the mechanical properties of bone [5].

The ovariectomized rat can be an appropriate experimental model to study the effects of postmenopausal osteoporosis treatments on bone.

The aim of this work was to study the effects of treatments to ovariectomized rats at a long range with a bisphosphonate (ZA) and with strontium ranelate. Treatments were administered immediately after ovariectomy and over a period of 8 months. Subsequently, BMD, trabecular and cortical microstructure, mineralization and biomechanics will be studied.

\section{Materials and Methods}

Sixty 6-month-old female Wistar rats weighing $325 \pm 54 \mathrm{~g}$ (mean $\pm \mathrm{SD}$ ) were used in this study. The animals were kept under constant living conditions $\left(22^{\circ} \mathrm{C}, 12\right.$-hour light-dark cycles per day), and food (standard laboratory chow) and water were available ad libitum.

The animals were randomized into the following groups: SHAM $(n=15)$, simulated intervention; OVX $(n=15)$, ovariectomized; OVX+ZA $(\mathrm{n}=15)$, ovariectomized and treated with ZA $(0.083 \mathrm{mg} / \mathrm{kg}$ i.v, 1 time the first day of treatment); OVX $+\operatorname{SrR}(n=15)$ ovariectomized treated with $\operatorname{SrR}(0.033$ $\mathrm{g} / \mathrm{kg} /$ day by oral gavage). Treatments started 1 day after ovariectomy and were maintained over 8 months.

One day after the last treatment, the experimental animals were weighed and killed by exsanguination under ether anesthesia. Once the blood was collected, the animals were frozen at $-20^{\circ} \mathrm{C}$ until determination of bone mineral density (BMD) in previously thawed animals. Prior to BMD analyses, the left femurs were excised and cleaned of adjacent tissue for BMD determination and computerized microtomography (micro-CT) and biomechanical testing. The right femur was also excised and cleaned for Raman studies. Lumbar spine BMD was determined in situ. Repeated freeze-thaw cycles have been shown to not influence the mechanical properties of bone [6]. All procedures were carried out in accordance with European Community standards on the care and use of laboratory animals and after approval of the Ethics Committee of Instituto de Investigación Sanitaria Fundación Jiménez Díaz.

\subsection{Bone Mineral Density}

BMD was determined in situ in the lumbar spine (L2, L3, and L4) and in the entire right femur by dual-energy x-ray densitometry (DEXA) using a HOLOGIC QDR-1000 TM (S/N 277) (Hologic, Inc., Waltham, MA, USA) with smallanimal software [7]. Intra- and inter-assay coefficients of variation $(\mathrm{CV})$ were $<0.53 \%$ and $<1.2 \%$, respectively. The scans of the femur were analyzed to determine the BMD of the whole femur. The scans of the L2, L3, and L4 vertebrae were analyzed for BMD and the results were expressed as the mean of the values obtained.

\subsection{Trabecular and Cortical Microarchitecture Analysis of Femur by Micro-CT}

The distal region of the left femur was analyzed by micro-CT (Skyscan N.V., Aartselaar, Belgium), imaged with an x-ray tube voltage of $50 \mathrm{kV}$ and a current of $200 \mu \mathrm{A}$ and with a $0.5-\mathrm{mm}$ aluminum filter. The scanning angular rotation was $185^{\circ}$ and the angular increment was $0.40^{\circ}$. The nominal resolution was $8 \mu \mathrm{m}$. Data sets were reconstructed using a modified Feldkamp algorithm [8]. For analysis of the microarchitectural properties of trabecular and cortical bone regions, femora specimens were evaluated within a conforming volume of interest (VOI). Both trabecular and cortical bone regions were obtained by free-drawing regions of interest and analyzed using the commercial software provided with the equipment from the microtomography data sets (integrated over a VOI) using direct 3D morphometry. Total volume of VOI (tissue volume (TV), $\left.\mathrm{mm}^{3}\right)$ and trabecular bone volume ((BV), $\mathrm{mm}^{3}$ ) were calculated based on the hexahedral marching cubes volume model of the VOI. Trabecular BV/TV (\%) was calculated directly. Trabecular thickness (Tb. Th; $\mu \mathrm{m})$, trabecular separation (Tb. Sp; $\mu \mathrm{m})$, and trabecular number (Tb. N; $1 / \mathrm{mm}$ ) were measured directly on 3D images using methods previously described [9, 10]. Measurements of trabecular thickness were calibrated by scanning and analyzing 3 
aluminum foils with thicknesses of 50, 125, and $250 \mu \mathrm{m}$. The non-metric indices, structure model index (SMI), and the connectivity density (Conn. D) were also calculated using the direct 3D model. The SMI parameter indicates the relative prevalence of rods and plates in a 3D structure [11]. It is a dimensionless parameter. The Conn. D is an index of the number of connections between trabecular structures by volume unit, expressed in $\mathrm{mm}^{3}$ [12].

Cortical bone parameters were measured in $2 \mathrm{D}$ from individual 2D cross-sectional images. A total of 497 slices were analyzed, starting at a distance of $6.00 \mathrm{~mm}$ from the growth plate, and extending a further longitudinal distance of $2.00 \mathrm{~mm}$. The following parameters were measured in the cortical region:

-Total cross-sectional area inside the periosteum, Tt. Ar: the mean value of the area occupied by bone and bone marrow in the transversal sections analyzed. It is expressed in $\mathrm{mm}^{2}$.

-Cortical bone area, Ct. Ar: the mean value of the area occupied by bone in the transversal sections analyzed. It is expressed in $\mathrm{mm}^{2}$.

-Marrow area, Ma. Ar: the mean value of the area occupied by bone marrow in the transversal sections analyzed. It is expressed in $\mathrm{mm}^{2}$.

-Cortical bone area fraction, Ct. Ar/Tt. Ar: this parameter indicates the percentage of total area occupied by bone, resembling BV/TV in trabecular bone.

-Cortical thickness, Ct. Th: a calculation of the thickness of the cortical region.

-Endosteal perimeter, Ec. Pm: the perimeter of the inner side of the cortical wall.

-Periosteal perimeter, Ps. Pm: the perimeter of the outer side of the cortical wall.

-Polar moment of inertia, $\mathrm{J}$ : indicates the resistance to rotation of a cross-section about a chosen axis being the rotational analogue of mass for linear motion. The $\mathrm{CV}$ values for all these measurements were $<5 \%$.

\section{Results}

\subsection{Bone Mineral Density}

\section{FBMD}

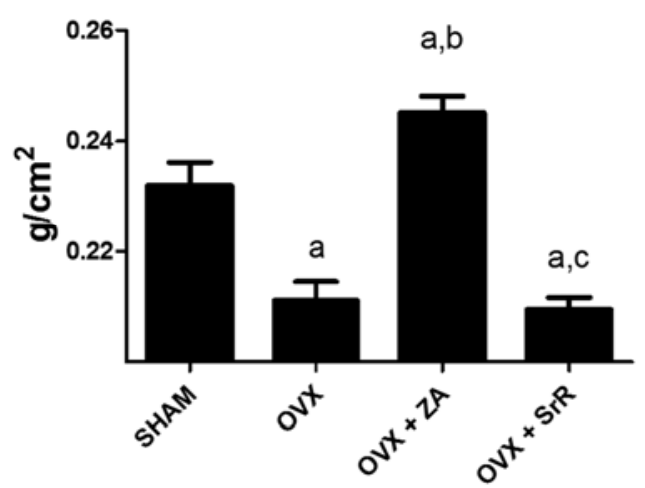

\subsection{Biomechanical Testing}

The femora underwent mechanical testing with a Microtest EM1/10/FR/m testing machine (Microtest, S. A., Madrid, Spain), and 3-point bending strength was measured. Each bone was compressed with a constant speed of $10 \mathrm{~mm} / \mathrm{min}$ until failure. A load-displacement curve was obtained and used to achieve bone structural parameters (extrinsic). The load-displacement curve was normalized and converted into a stress-strain curve to obtain bone material properties (intrinsic) [13]. The extrinsic biomechanical properties of whole bone reported include ultimate load (maximum force that the specimen sustained), stiffness (the slope of the linear portion of the load-displacement curve), and work to failure (the area under the load-displacement curve). In addition, bone tissue intrinsic biomechanical properties include ultimate stress, apparent Young's modulus and toughness, which are independent of cross-sectional size and shape.

\subsection{Raman Spectrometry}

The middle-cortical zone of the right femur was analyzed by Raman spectroscopy with a Horiba Jobin Yvon's LABRam micro-Raman spectrophotometer. Three functional groups were detected: $\mathrm{PO}_{4}{ }^{3-}, \mathrm{CO}_{3}{ }^{2-}$, and an amide group. From the peaks of these functional groups, 3 indexes were determined: crystallinity index (CI), a wide of band at the middle height of phosphate; mineralization index (MI), phosphate band intensity/amide band intensity; substitution index (SI), phosphate band intensity/carbonate band intensity.

\subsection{Statistical Analysis}

The results of the experiments were expressed as the mean \pm SD of the different parameters. A non-parametric method, the Mann-Whitney test (Medcalc Software Program, Belgium), was used to compare the different treatment groups. A p-value $<0.05$ was accepted as denoting a significant difference. 
As compared with the SHAM group, femoral BMD (FBMD) and lumbar BMD (LBMD) were significantly decreased in OVX rats $(\mathrm{p}<0.0001$ and $\mathrm{p}<0.0001$, respectively). Administration of $\mathrm{SrR}$ to rats did not produce any significant change in FBMD or in LBMD with respect to the OVX group. Administration of ZA prevented the decrease in FBMD ( $p<0.001$ vs OVX group) and LBMD ( $p<0.01$ vs OVX group) due to ovariectomy, and BMD levels were higher than those of the SHAM group (Fig. 1).

\subsection{Trabecular Microarchitecture}

Figure 2 shows bone microstructural parameters. Eight months after surgery, most of the variables characterizing

\section{BV/TV}

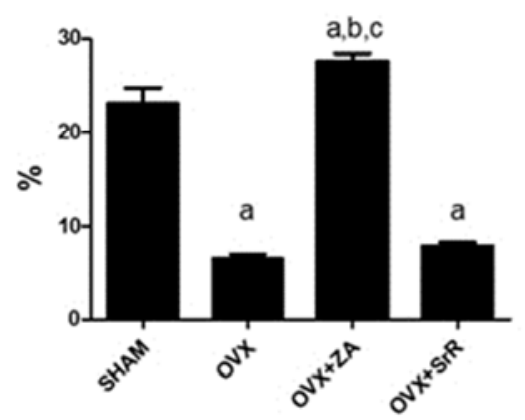

Tb.N
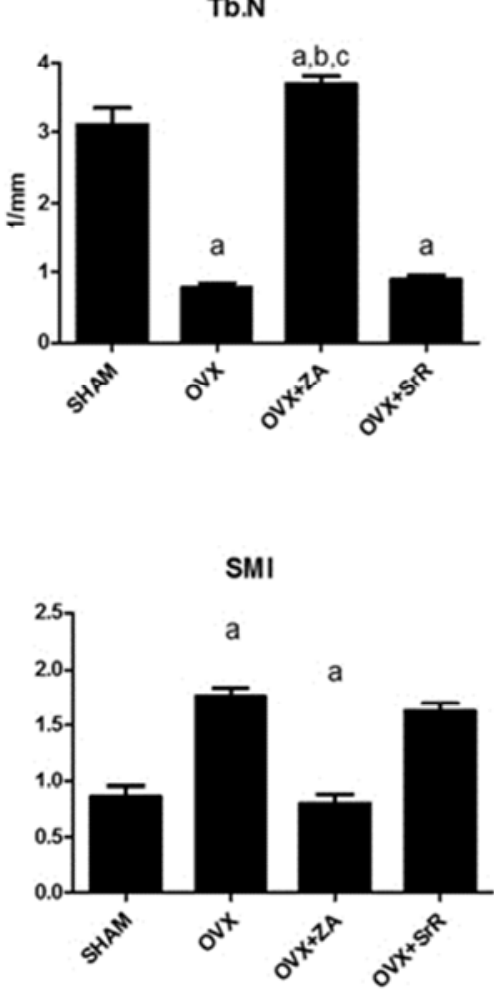

bone structure from micro-CT were significantly different in the OVX group with respect to the SHAM group. OVX rats presented a significant decrease in $\mathrm{BV} / \mathrm{TV}$ in the distal femora $(p<0.05)$. This decrease in BV/TV was due to a significant increase in $\mathrm{Tb}$. $\mathrm{Sp}$ and a decrease in $\mathrm{Tb} . \mathrm{N}$ and $\mathrm{Tb}$. Th. The administration of SrR did not prevent the changes from occurring in OVX, showing similar values of BV/TV, Tb. Sp, Tb. N, and Tb. Th to those obtained in the OVX group. The administration of ZA prevented all of the changes that had occurred in OVX, producing similar values of BV/TV, Tb. Th, and Tb. Sp to those obtained in the SHAM group and higher levels of $\mathrm{Tb} . \mathrm{N}$ in treated rats than in the SHAM group.
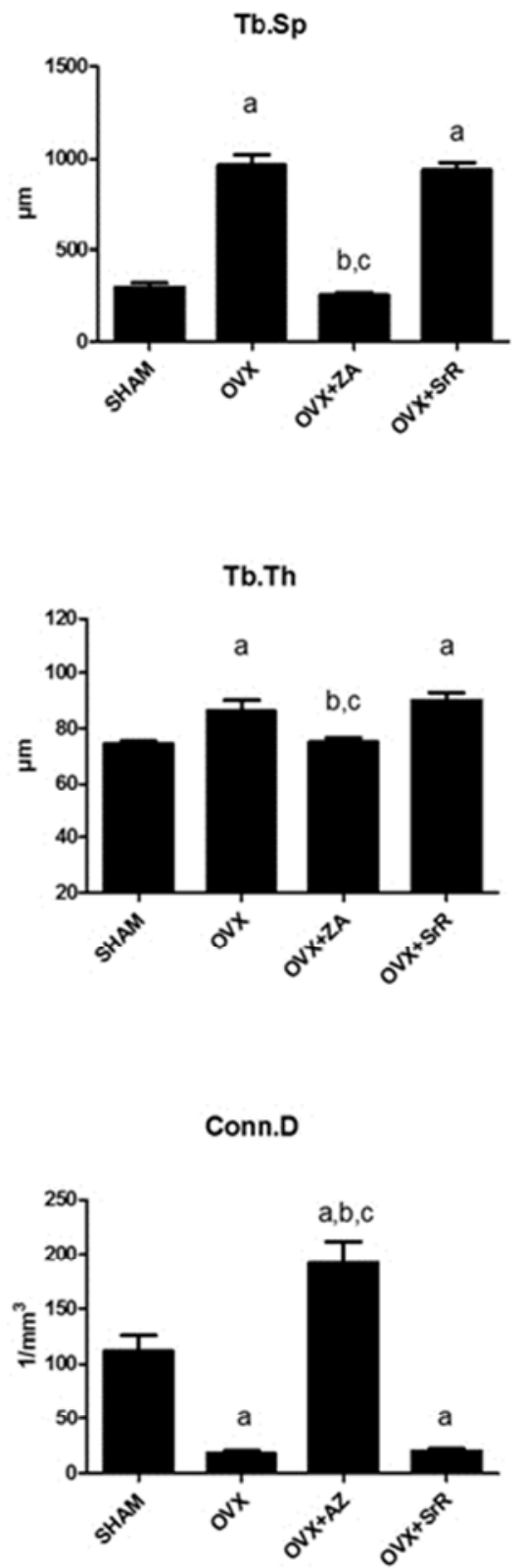

Figure 2. Femoral trabecular microarchitecture analysis: bone volume/total volume (BV/TV), trabecular separation (Tb.Sp), trabecular number (Tb.N), trabecular thickness (Tb.Th), structure model index (SMI), and connectivity density (Conn.D). Sixty 6-month-old female Wistar rats at the beginning of the study, distributed into 4 groups: SHAM ( $n=15)$, simulated intervention; OVX $(n=15)$, ovariectomized; OVX+ZA ( $n=15)$, ovariectomized and treated for 8 months with ZA (0.083 mg/kg i.v., one time the first day of treatment); OVX+SrR ( $=15)$ ovariectomized and treated for $8 \mathrm{months}$ with SrR (0.033 g/kg/day by oral gavage). Data are expressed as mean \pm SD. Statistical significance $p<0.05$ : (a) vs. SHAM; (b) vs. OVX; (c) vs. OVX+SrR. 
Ovariectomy also induced significant changes in nonmetric parameters. SMI was significantly increased due to surgery, indicating a transformation of trabecular bone from a plate- to a rod-like structure. ZA treatment preserved SHAM levels but no differences were observed with respect to the OVX group with SrR treatment. Conn. D also decreased in OVX group, indicating a decrease in the number of connections in trabecular structure. In comparison with the OVX group, rats treated with ZA showed a significant increase in this parameter even with respect SHAM group. SrR treatment did not produce any difference with untreated OVX rats (Figure 2).

Figure 3 shows a representative image of bone trabecular microarchitecture in all the studied groups.

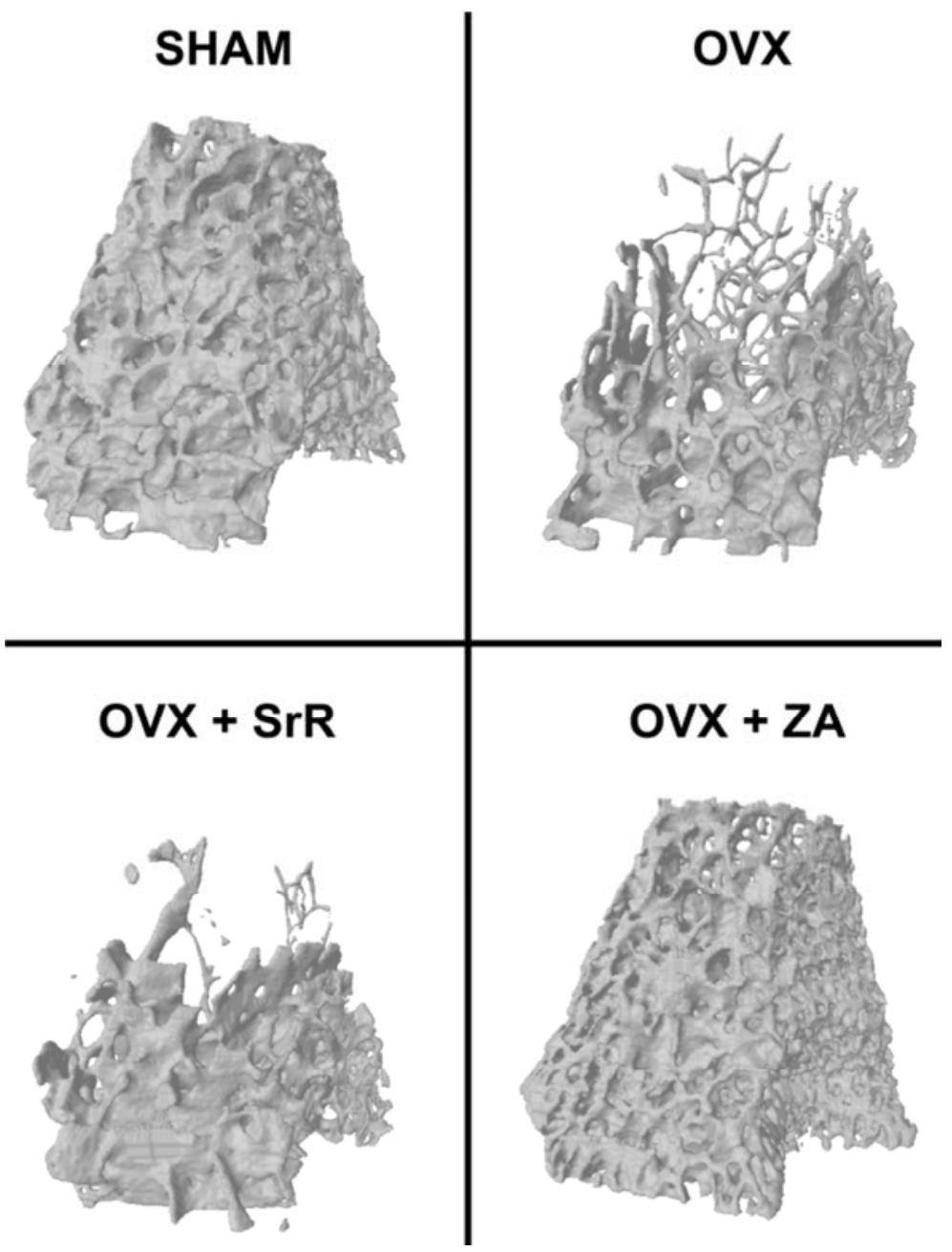

Figure 3. Representative images of bone trabecular 3D microarchitecture in femur sections obtained by computed microtomography. Study formed by SHAMoperated rats (SHAM), castrated rats (ovariectomized: OVX), and castrated rats treated for 8 months with SrR (0.033 g/kg/day by oral gavage) (OVX+ SrR) or castrated rats treated for 8 months with ZA $(0.083 \mathrm{mg} / \mathrm{kg}$ i.v, one time the first day of treatment) $(O V X+Z A)$.

\subsection{Cortical Microarchitecture}

Table 1 shows the microstructural parameters of the cortical region of the femurs of the studied groups. There was no modification in any of the studied groups with respect to the SHAM group. (See Table 1 at the end of this work)

Table 1. Micro-CT cortical properties applied to the femur of ovariectomized rats treated with ZA and SrR.

\begin{tabular}{lllllllll}
\hline & Tt. $\mathbf{A r} \mathbf{~ m m}^{2}$ & Ct. $\mathbf{A r} \mathbf{~ m m}^{2}$ & Ma. $\mathbf{A r} \mathbf{~ m m}^{2}$ & Ct. Ar/Tt. Ar\% & Ct. Th $\boldsymbol{\mu m}$ & Ps. Pm $\mathbf{~ m m}$ & $\mathbf{E c . ~ P m ~} \mathbf{~ m m}$ & $\mathbf{J ~ m m}$ \\
\hline SHAM & $10,48 \pm 0,81$ & $5,80 \pm 0,36$ & $4,63 \pm 0,52$ & $55,43 \pm 2,07$ & $585,74 \pm 25,03$ & $12,66 \pm 0,53$ & $10,59 \pm 1,39$ & $15,01 \pm 2,27$ \\
OVX & $10,99 \pm 1,08$ & $6,02 \pm 0,40$ & $4,95 \pm 0,74$ & $54,96 \pm 2,91$ & $602,22 \pm 19,37$ & $12,98 \pm 0,61$ & $9,30 \pm 0,70$ & $16,52 \pm 2,73$ \\
SrR & $11,04 \pm 0,93$ & $5,96 \pm 0,23$ & $5,07 \pm 0,73$ & $54,15 \pm 2,82$ & $591,42 \pm 20,15$ & $13,03 \pm 0,64$ & $9,51 \pm 0,84$ & $16,48 \pm 2,44$ \\
ZA & $10,41 \pm 0,82$ & $6,01 \pm 0,16$ & $4,35 \pm 0,71$ & $58,00 \pm 3,63$ & $625,72 \pm 25,60$ & $12,52 \pm 0,46$ & $9,51 \pm 1,12$ & $14,89 \pm 1,73$ \\
\hline
\end{tabular}

Sixty 6-month-old female Wistar rats, sham-operated (SHAM), ovariectomized (OVX), OVX and treated with zoledronic acid (ZA) (0.08mg/kg i.v), and OVX and treated with strontium ranelate $(\mathrm{SrR})(0.033 \mathrm{~g} / \mathrm{kg} / \mathrm{day}$ v. o) for 8 months Tt. Ar: Total cross-sectional area inside the periosteum; Ct. Ar: Cortical bone area; Ma. Ar: Marrow area; Ct. Ar/Tt. Ar: Cortical bone area fraction; Ct. Th: Cortical thickness; Ec. Pm: Endosteal perimeter; Ps. Pm: Periosteal perimeter; J: Polar moment of inertia. Statistical significance: Mann-Whitney test. No significant statistical differences between groups were observed. 
Fig. 4 shows a representative image of the cortical bone of the SHAM, SrR, and ZA groups.

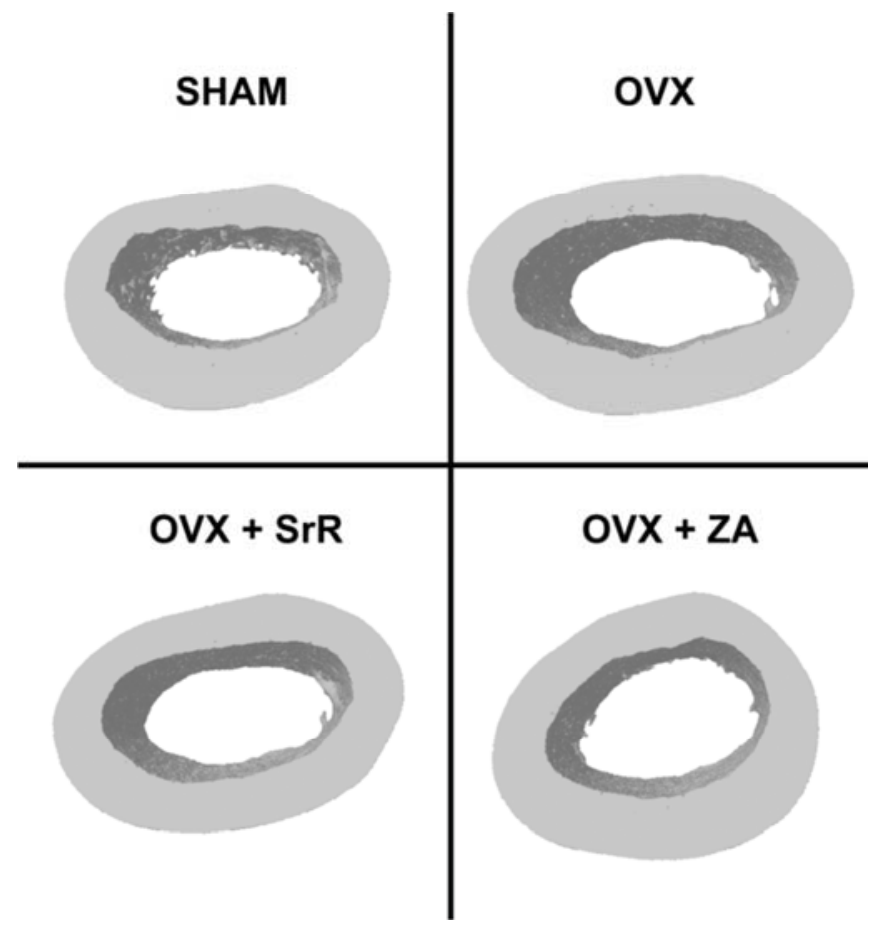

Figure 4. Representative images of cortical microarchitecture measured in $2 D$ from individual $2 D$ cross-sectional images. Study formed by SHAM-operated rats (SHAM), castrated rats (ovariectomized: OVX) and castrated rats treated for 8 months with $\mathrm{SrR}(0.033 \mathrm{~g} / \mathrm{kg} /$ day by oral gavage) (OVX+SrR) or castrated rats treated for 8 months with $Z A(0.083 \mathrm{mg} / \mathrm{kg}$ i.v., one time, the first day of treatment) $(O V X+Z A)$.

\subsection{Biomechanical Testing}

Table 2 shows the extrinsic (structural) and intrinsic (material) biomechanical properties of the femurs of the 4 groups of rats studied. Ovariectomy produced a significant decrease in extrinsic stiffness and apparent Young's modulus, and this decrease was maintained in spite of treatment with SrR. ZA treatment produced an increase in ultimate load, extrinsic stiffness, ultimate stress, and work to failure, and these values were even higher than those of the SHAM group. (See Table 2 at the end of this work)

Table 2. Extrinsic (structural) and intrinsic (material) biomechanical properties of the femur of ovariectomized rats treated with ZA and SrR.

\begin{tabular}{|c|c|c|c|c|c|}
\hline & & \multicolumn{4}{|c|}{ Extrinsic properties (structural) } \\
\hline & & SHAM & OVX & $\mathrm{OVX}+\mathrm{ZA}$ & $\mathrm{OVX}+\mathrm{SrR}$ \\
\hline Ultimate load, $\mathrm{N}$ & $\mathrm{F}_{\text {ult }}$ & $140.66 \pm 21.35$ & $143.53 \pm 9.16$ & $163.86 \pm 23.44^{\mathrm{a}, \mathrm{b}, \mathrm{c}}$ & $150.32 \pm 11.95$ \\
\hline Extrinsic stiffness, $\mathrm{N} / \mathrm{mm}$ & $\mathrm{S}$ & $151.58 \pm 14.35$ & $135.85 \pm 16.49^{\mathrm{a}}$ & $169.83 \pm 9.88^{\mathrm{b}, \mathrm{c}}$ & $138.9 \pm 9.14^{\mathrm{a}}$ \\
\hline \multirow[t]{3}{*}{ Work to failure, $\mathrm{mJ}$} & $\mathrm{U}$ & $75.22 \pm 23.75$ & $91.2 \pm 17.22$ & $84.98 \pm 19.01$ & $95.00 \pm 12.93$ \\
\hline & & \multicolumn{4}{|c|}{ Intrinsic properties (material) } \\
\hline & & SHAM & ovX & $\mathrm{OVX}+\mathrm{ZA}$ & $\mathrm{OVX}+\mathrm{SrR}$ \\
\hline Ultimate stress, $\mathrm{MPa}$ & $\sigma_{\text {ult }}$ & $173.96 \pm 27.17$ & $164.74 \pm 25.49$ & $197.55 \pm 29.96^{\mathrm{a}, \mathrm{b}, \mathrm{c}}$ & $176.54 \pm 14.52$ \\
\hline Apparent Young's modulus, MPa & $\mathrm{E}$ & $2484.8 \pm 386.56$ & $2053.98 \pm 642.16^{\mathrm{a}}$ & $2673.09 \pm 473.80^{\mathrm{b}, \mathrm{c}}$ & $2107.13 \pm 248.09^{\mathrm{a}}$ \\
\hline Toughness, MPa & $\mathrm{u}$ & $7.46 \pm 2.88$ & $8.05 \pm 1.01$ & $7.93 \pm 1.77$ & $8.66 \pm 1.13$ \\
\hline
\end{tabular}

Sixty 6-month-old female Wistar rats, sham-operated (SHAM), ovariectomized (OVX), OVX and treated with ZA (0.08mg/kg i.v.) (OVX+ZA) and OVX and treated with $\mathrm{SrR}(0.033 \mathrm{~g} / \mathrm{kg} /$ day v.o.) $(\mathrm{OVX}+\mathrm{SrR})$ for 8 months. Data are expressed as mean \pm SD. Statistical significance $\mathrm{p}<0.05$ : (a) vs. SHAM; (b) vs. OVX; (c) vs. OVX+SrR.

\subsection{Raman Spectrometry}

Figure 5 shows a representative Raman spectrum corresponding to a rat of the SHAM group. Functional groups $\left(\mathrm{PO}_{4}{ }^{3-}, \mathrm{CO}_{3}{ }^{2-}\right.$, and amide) can be observed. Results were similar in all the groups. Figure 6 shows the values of SI, CI, and MI in the 4 groups of studied rats. With respect to
SI, there were no significant differences between the studied groups. Ovariectomy did not produce significant differences in crystallinity, and the values of this parameter were higher in groups $\mathrm{SrR}$ and $\mathrm{ZA}$, although without significant differences. MI decreased significantly in the OVX group. Treatment with SrR was not able to prevent this effect, though rats treated with ZA presented MI values that were 
similar to those of SHAM group.

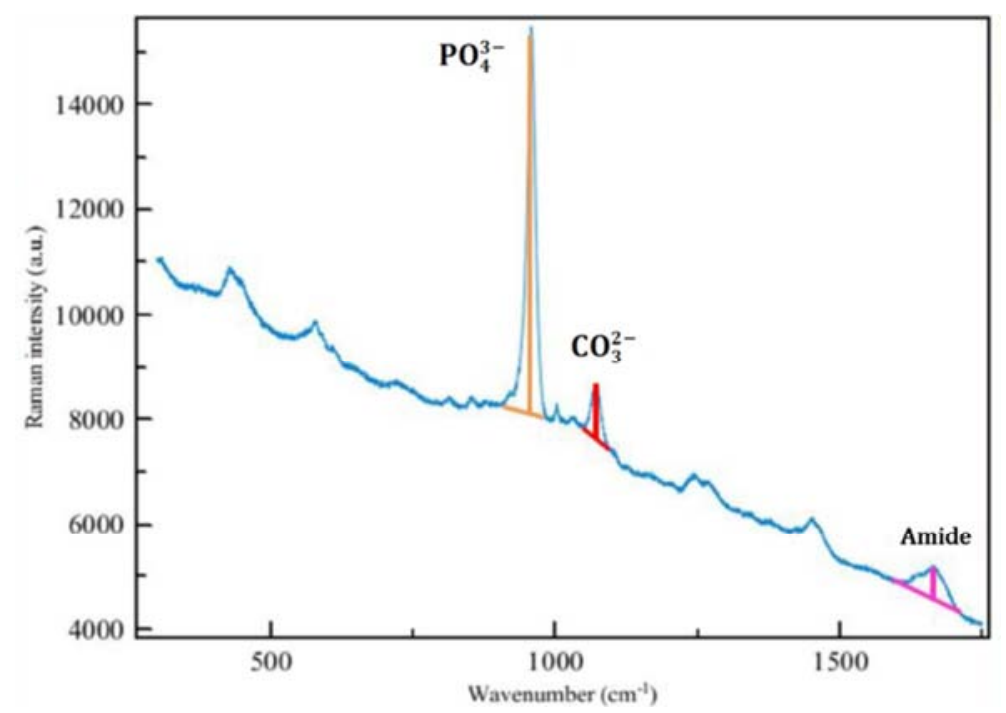

Figure 5. Representative Raman spectrum corresponding to a rat of the SHAM group. Functional groups $\left(\mathrm{PO}_{4}^{3-}, \mathrm{CO}_{3}{ }^{2-}\right.$ and amide) can be observed.

Mineralization

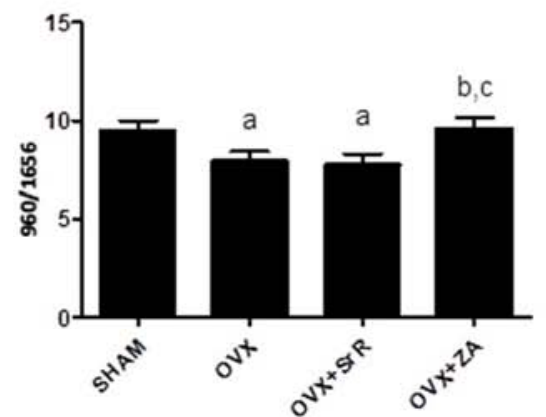

Substitution index

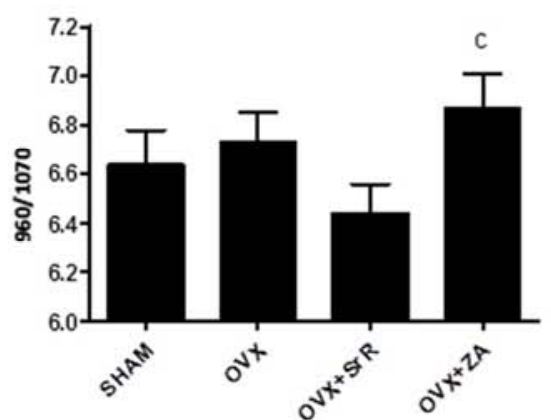

Crystallinity

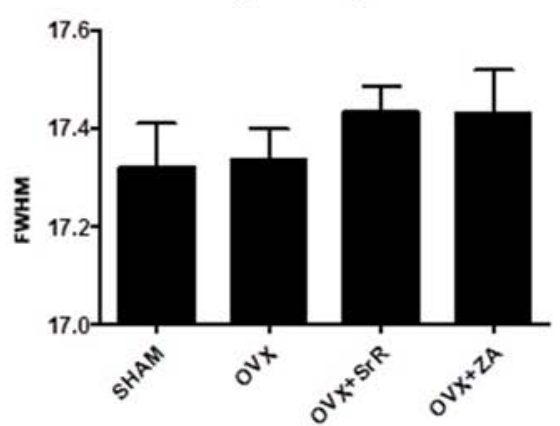

Figure 6. Values of crystallinity index (CI), mineralization index (MI) and substitution index (SI) derived from the Raman spectrum of the middle-cortical zone of femur. 960/1656: Raman intensity (a.u.) of phosphate band (960 cm-1)/ Raman intensity (a.u.) of amide band (1656 cm-1)x100; 960/1070: Raman intensity (a.u.) of phosphate band (960 cm-1)/ Raman intensity (a.u.) of carbonate band (1070 cm-1)x100. FWHM: Peak's full width at half maximum in phosphate band. Sixty 6-month-old female Wistar rats at the beginning of the study, distributed into 4 groups: SHAM ( $n=15)$, simulated intervention; OVX ( $n=15)$, ovariectomized; $O V X+Z A(n=15)$, ovariectomized and treated for 8 months with $Z A(0.083 \mathrm{mg} / \mathrm{kg}$ i.v., one time the first day of treatment); OVX+SrR ( $=15)$ ovariectomized and treated for 8 months with $S r R(0.033 \mathrm{~g} / \mathrm{kg} /$ day by oral gavage). Data are expressed as mean $\pm S D$. Statistical significance $p<0.05:$ (a) vs. SHAM; (b) vs. OVX; (c) vs. OVX+SrR.

\section{Discussion}

Postmenopausal osteoporosis is a chronic disease that exerts a significant burden on both individuals and the community. Hence, there is a need for long-term treatment to be associated with a positive benefit-risk balance.

Strontium ranelate, a therapeutic option for osteoporosis, was thought to have a dual mode of action, simultaneously stimulating bone formation and reducing resorption [14]. ZA, a bisphosphonate, is a potent antirresorptive agent [2]. The present work shows that long-term (8 months) treatment with these 2 agents produce different effects on BMD in OVX rats. While $\mathrm{ZA}$ treatment prevented losses of BMD due to ovariectomy and moreover led to a more compact bone than that of the SHAM group, the effects of SrR were not detectable in the BMD of OVX rats.

Similarly, with respect to bone microstructural parameters, in our study SrR administration did not prevent changes occurring after ovariectomy that are related to a decrease in $\mathrm{BV} / \mathrm{TV}, \mathrm{Tb}$. N, and the Tb. Th and to an increase in Tb. Sp. The administration of ZA totally prevented these changes while producing higher levels of $\mathrm{Tb} . \mathrm{N}$ than in the SHAM group. A similar effect was observed in the non-metric parameters SMI and Conn. D.

With respect to the effects of SrR on bone quality in ovariectomized rats, there are controversial data in the literature. Chen et al. [15] found that the administration of SrR $(500 \mathrm{mg} / \mathrm{kg} /$ day) to 3-month-old ovariectomized rats during 12 weeks increased femoral BMD, distal femoral bone histomorphometric parameters, and biomechanical parameters when compared to the OVX group. The dose of SrR used by these authors is 15 times higher than ours and this could be the cause of the different effects of SrR observed on OVX rats.

In another work, Chen et al. [16], using a similar dose of 
SrR to that of the previously mentioned work (500 $\mathrm{mg} / \mathrm{kg}$ /day orally for 12 weeks), obtained a significant increase in distal femoral BMD when compared with the OVX group. These authors also administered ZA to a group of OVX rats $(0.1 \mathrm{mg} / \mathrm{kg}$ i.v, a single injection). They observed that ZA increased BMD significantly more than SrR. They also studied osseointegration of bone implants where SrR was inferior to groups ZA and SHAM in bone-toimplant contact and peri-implant bone fraction. Boyd et al. [17] administered a diet containing $\operatorname{SrR}(900 \mathrm{mg} / \mathrm{kg} /$ day $)$ for a period of 104 weeks to intact female Fischer rats. SR produced a significant improvement in bone microarchitecture compared to controls, including a $40 \%$ higher trabecular thickness, 28\%-higher cortical thickness, and no significant change in the number of trabeculae. The authors' use of finite element analysis resulted in greater bone strength under treatment with strontium ranelate. The dose of SrR used in this work is higher than ours, and the duration of the treatment is also higher (104 weeks vs 8 months). With higher doses of SrR than ours $(625 \mathrm{mg} / \mathrm{kg} /$ day vs $33 \mathrm{mg} / \mathrm{kg} /$ day) administered over 5 to 7 days, Zacchetti et al. [18] demonstrated that treatment with SrR accelerates the filling of a bone defect and improves the material level properties of the healing bone. Ozturan et al. [19] also demonstrated that $\mathrm{SrR}$ at $450 \mathrm{mg} / \mathrm{kg}$ /day over 6 weeks accelerates fracture healing in OVX rats. Yalin et al. [20] observed that $\mathrm{SrR}$ has a preventive effect on oxidative damage in ovariectomized rats starting 4 months after ovariectomy. Using a lower dose of SrR (0.026-0.105 $\mathrm{mg} / \mathrm{kg} /$ day) that was similar to the one used in our work, Marie et al. found that over a period of 6 months this treatment prevented trabecular bone loss.

As in our study, other authors did not find effects of SrR on bone. Ma et al. [22], administering 150 or $450 \mathrm{mg} / \mathrm{kg} /$ day of SrR to 6-month-old OVX rats, found that this compound did not enhance bone strength and had no effect on histomorphometry of the tibial metaphysis. Brüel et al. [23] did not find any effect of SrR on immobilization-induced bone loss in a rat model in which immobilization was induced by injecting botox into the muscles of the upper limbs.

In the case of treatment with $\mathrm{ZA}$, we did not find controversial results in the literature. Khajuria et al. [24], using a ZA dose of $50 \mu \mathrm{g} / \mathrm{kg}$ in ovariectomized 3-month-old female rats 12 weeks post-surgery and over a period of 12 weeks thereafter, observed a restoration of BMD and cortical and trabecular bone microarchitecture.

In an interesting article, Brennan et al. [25] quantified the distribution parameters of BMD in trabeculae from the proximal femora of an ovariectomized sheep model that underwent estrogen deficiency for 31 months and after receiving ZA. These authors demonstrated that prolonged estrogen deficiency leads to decreased mineralization and increased heterogeneity. Interestingly, ZA treatment of OVX sheep significantly reduced tissue mineral variability, both at a trabecular level and between femoral regions. These findings indicate that $\mathrm{ZA}$ treatment acts to reverse the increased mineral heterogeneity occurring during estrogen deficiency.

Palacio et al. [26] showed that $0.1 \mathrm{mg} / \mathrm{kg}$ of ZA administered intraperitoneally to female ovariectomized rats (2 months old) significantly increased biomechanical parameters (maximum load and yield point) and reduced the stiffness coefficients in the femurs of the rats.

$\mathrm{Wu}$ et al. [27] administered zoledronate-impregnated calcium phosphate cement (ZLN/CPC) to OVX rats. The animals were implanted with ZLN/CPC containing $0.025 \mathrm{mg}$ of ZLN in the greater cementum. After 8 weeks, micro-CT scanning and histological examinations of the distal femoral metaphyses showed that the cancellous bone architectures were restored with a concomitant decrease in bone porosity. The bone mineral content in the bone ashes also increased significantly.

The biomechanical properties of the femurs of treated rats reflect the findings in terms of BMD and bone microstructure as those of rats treated with $\mathrm{SrR}$ were similar to those of ovariectomized rats and those of rats treated with ZA were even better than those of SHAM group. The results of Palacio et al. [26] agree with our findings, reporting that administration of ZA to OVX rats $(0.1 \mathrm{mg} / \mathrm{kg}$ intraperitoneally) significantly increased the maximum load and yield point. Also, according to our results, Ma et al. [22] found that SrR did not enhance bone strength relative to agematched ovariectomized controls.

With respect to the Raman study, in this work we did not find significant differences in the $\mathrm{CI}$ and substitution (carbonates/phosphates) index between SHAM, OVX, and treated groups. However, ovariectomy decreased the mineralization index (MI), and these levels only recovered with ZA treatment.

Querido et al. [28] evaluated the effects of SrR on the composition of the crystal structure of biological bone-like apatite produced in osteoblast cell cultures. In the study, cells were treated with $\mathrm{SrR}$ at concentrations of 0.05 and $0.5 \mu \mathrm{M}$ $\mathrm{Sr}^{2+}$. These authors found several changes in the mineralization produced under $0.5 \mu \mathrm{M} \mathrm{Sr}^{2+}$, such as an increase in $\mathrm{CO}_{3}{ }^{2-}$ substitutions and changes in $\mathrm{Ca} / \mathrm{P}$ ratio. They did not find these changes under $0.05 \mu \mathrm{M}$ Sr2+ conditions. In the present work, we did not find changes in $\mathrm{Ca} / \mathrm{P}$ ratio or carbonates/phosphates index, possibly because our treatment does not reach a concentration of $0.5 \mu \mathrm{M} \mathrm{Sr}^{2+}$. Rossi et al. [29] also found an increase in the number of carbonate groups in the evaluation of apatite for the mandibles of rats treated with $\mathrm{SrR}$ in drinking water. The concentration of $\mathrm{SrR}$ consumed by the animals compared to our experiment cannot be established.

In a study performed in humans, Doublier et al. [30] compared biopsies of osteoporotic women treated with $\mathrm{SrR}$ or placebo, finding that the quality of bone apatite crystals was maintained without changes in crystallinity after 36 months of treatment with SrR.

In accordance with the results reported here, Gamsjaeger et al. [31], using Raman iliac-crest biopsies from the HORIZON-PFT clinical trial, found that once-yearly 
administration of intravenous ZA for 3 years in humans did not exert any adverse effects on the evolution of bone material properties.

It is important to emphasize that the concentrations of the drugs used in this work were weight $(\mathrm{kg})$-adjusted to match those used to treat humans (SrR $2 \mathrm{~g} /$ day, ZA $5 \mathrm{mg} /$ year). With respect to the administration and distribution of strontium in rat bone, Dahl et al. [32] found that after repeated administration over the previous 4 weeks, strontium ranelate reaches a plateau. After treatment withdrawal, strontium exchanged onto crystals is rapidly eliminated, but not during administration.

As mentioned, throughout this discussion there are discrepancies in the results found by the different authors after treatment of ovariectomized rats with SrR and these differences can be positive or negative. With respect to the studies in humans, in the clinical trials SOTI and TROPOS [33] extended to 10 years, positive effects of SrR on BMD and fracture risk are found. However, there are also authors who reported negative results for treatment with $\mathrm{SrR}$ in humans [34].

Due to the fact that in this work we present a long range treatment ( 8 months in the life of rats), we cannot rule out the possibility that SrR had a positive effect on OVX rats during the first phase, but that over a longer period of time the treatment did not prevent the effects of ovariectomy.

With respect to treatment with ZA, both, works carried out using ovariectomized animals and those conducted in patients, the most important the clinical trial, called the HORIZON Pivotal Fracture Trial [35], a 10-year trial, showed the positive effects of this treatment on bone. The findings of this trial revealed that the BMD of treated patients was higher than that of controls, thus reflecting our work with ovariectomized rats.

In conclusion, the results of the present work show that preventive treatment with $\mathrm{SrR}$ at a concentration of 0.033 $\mathrm{g} / \mathrm{kg} /$ day for 8 months did not revert the alterations in bone quality due to ovariectomy in rats; however, it cannot be ruled out that SrR made in a first phase a positive effect on OVX rats, although when administered over a longer period of time it did not prevent the effects of ovariectomy. On the other hand, treatment with ZA $(0.083 \mathrm{mg} / \mathrm{kg}$,i.v., single injection) not only reverted the effects of the estrogen deficiency, but also improved bone quality with respect to control rats.

\section{Acknowledgements}

This work was supported by a grant of the "Instituto de Salud Carlos III”, Spain, PI12/01472, FEDER.

\section{References}

[1] Wariner AH, Curtis JR. Adherence to osteoporosis treatments: room for improvement. Curr Opin Rheumatol 2009 21: 356-62.
[2] Lyles KW, Colón-Emeric CS, Magaziner JS, Adachi JD, Mautalen C, Hyldstrup L, Recknor C, Nordsletten L, Moore KA, Lavecchia C, Zhang J, Mesembrink P, Hodson PK, Abrams K, Orloff JJ, Horowith Z, Eriksen FF, Boonen S . For the HORIZON recurrent fracture trial. N Eng J Med 2007; 357: 1799-1809.

[3] Reginster JY, Bruyére O, Sawicki A, Roces-Varela A, Fardellone P, Roberts A, Devogelaer JP. Long-term treatment of postmenopausal osteoporosis with strontium ranelate: results at 8 years. Bone 2009; 45: 1059-64.

[4] Kleerekoper M. Osteoporosis prevention and therapy: preserving and building strength through bone quality. Osteoporos Int 2006; 17: 1707-15.

[5] Pistoia W, Van Rietbergen B, Rüegsegger P. Mechanical consequences of diferent scenarios for simulated bone athrophy and recovery in the distal radius. Bone 2003; 33: 937-45.

[6] Borchers RE Gibson LJ, Burchardt H, Hayes WC. Effects of selected thermal variables on the mechanical properties of trabecular bone. Biomaterials 1995; 16: 545-51.

[7] Gala Paniagua J, Díaz-Curiel M, De la Piedra Gordo C, CastillaReparaz C, TorralboGarcía M. Bone mass assessment in rats by dual energy X-ray absorptiometry. The British Journal of Radiology 1998; 71: 754-58.

[8] Feldkamp LA, Davis LC. Practical cone-beam algorithm. Journal of the Optical Society of America 1984 ; 1:612-19

[9] Hildebrand T, Ruegsegger P. A new method for the modelindependent assessment of thickness in three-dimensional images. Journal of Microscopy 1997;185: 67-75.

[10] Ulrich D, Van Rietbergen B, Laib A, Ruegsegger P. The ability of three-dimensional structural indices to reflect mechanical aspects of trabecular bone. Bone 1999; 25: 5560 .

[11] Hildebrand T, Rüegsegger P. Quantification of Bone Microarchitecture with the Structure Model Index. Computer Methods in Biomechanics and Biomedical Engineering 1997; $1: 15-23$.

[12] Odgaard A, Gundersen HJ. Quantification of connectivity in cancellous bone, with special emphasis on 3-D reconstructions. Bone 1993; 14: 173-182.

[13] Turner CH, Burr DB. Basic biomechanical measurements of bone: A tutorial. Bone 1993; 14: 595-608.

[14] Iolascon G, Frizzi L, Di Pietro G, Capaldo A, Luciano F, Gimigliano F. Bone quality and bone strength: benefits of the bone-forming approach. Clin Cases Miner Bone Metab 2014; 11: $20-4$.

[15] Chen B, Li Y, Yang X, Xie D. Comparable effects of alendronate and strontium ranelate on femur in ovariectomized rats. Calcif. Tissue Int 2013; 93: 481-86.

[16] Chen B, Li Y, Yang X, Xu H, Xie D. Zoledronic acid enhances bone-implant osseointegration more than alendronate and strontium ranelate in ovariectomized rats. Osteoporos. Int. $2013 ; 24,2115-21$.

[17] Boyd SK, Szabo E, Ammann P. Increased bone strength is associated with improved bone microarchitecture in intact female rats treated with strontium ranelate: A finite element analysis study. Bone 2011; 48, 1109-16. 
[18] Zacchetti G, RizZAi, Ammann P. Systemic treatment with strontium ranelate accelerates the filling of a critical size bone defect and improves the intrinsic quality of the healing bone. Bone 2012; 50, S57.

[19] Ozturan KE, Demir B, Yucel I, Cakici H, Yilmaz F, Haberal A. Effect of strontium ranelate on fracture healing in the osteoporotic rats. J. Orthop. Res. 2011; 29, 138-42.

[20] Yalin S, Sagir O, Comelekoglu U, Berkoz̈ M, Eroglu P. Strontium ranelate treatment improves oxidative damage in osteoporotic rat model. Pharmacol. Reports 2012; 64, 396402.

[21] Marie PJ, Host M, Modrowski D, De Poliak C, Guillemain J, Deloffre P. An uncoupling agent containing strontium prevents bone loss by depressing bone resorpion and maintaining bone formation in estrogen-deficient rats. J Bone Miner Res 1993 18: 547-51.

[22] Ma YL, Zeng QQ, Porras LL, Harvey A, Moore TL, Shelbourn TL, Dalsky GP, Wronsky TJ, Aguirre JI, Bryant HU, Sato M. Teriparatide [rhPTH (1-34)], but not strontium ranelate, demonstrated bone anabolic efficacy in mature, osteopenic, ovariectomized rats. Endocrinology 2011; 152, 1767-78.

[23] Brüel A, Vegger JB, Raffalt AC, Andersen JET, Thomsen JS. PTH (1-34), but not strontium ranelate counteract loss of trabecular thickness and bone strength in disuse osteopenic rats. Bone 2013; 53, 51-8.

[24] Khajuria DK, Razdan R, Mahapatra DR. Additive effects of zoledronic acid and propranolol on bone density and biochemical markers of bone turnover in osteopenic ovariectomized rats. Rev. Bras. Reumatol 2015; 55, 103-12.

[25] Brennan MA, Gleeson JP, O’Brien F J, McNamara LM. Effects of ageing, prolonged estrogen deficiency and Zoledronate on bone tissue mineral distribution. J. Mech.Behav. Biomed. Mater 2014; 29: 161-70.

[26] Palacio EP, Müller SS, Sardenberg T, MIzobuchi RR, Galbiatti JA, DUrigan A Jr, Savarese A, Ortolan EV. Detecting early biomechanical effects of zoledronic acid on femurs of osteoporotic female rats. J. Osteoporos 2012; 2012: 162806. doi 10.1155/2012/ 162806.
[27] $\mathrm{Wu}, \mathrm{CC}$, Wang $\mathrm{CC}, \mathrm{Lu} \mathrm{DH}$, Hsu LH, Yang KC, Lin FH. Calcium phosphate cement delivering Zoledronate decreases bone turnover rate and restores bone architecture in ovariectomized rats. Biomed. Mater 2012; 7: 035009.

[28] Querido W, Campos AP, Martins Ferreira EH, San Gil RA, Rossi AM, Farina M. Strontium ranelate changes the composition and crystal structure of the biological bone-like apatite produced in osteoblast cell cultures. Cell Tissue Res 2014; 357: 793-801.

[29] Rossi AL, Moldovan S, Querido W, Rossi A, Werckman J, ERsen O, Farina M. Effect of strontium ranelate on bone mineral: Analysis of nanoscale compositional changes. Micron 2014; 56: 29-36.

[30] Doublier A, Farlay D, Jaurand X, Vera R, Boivin GEffects of strontium on the quality of bone apatite crystals: A paired biopsy study in postmenopausal osteoporotic women. Osteoporos. Int. 2013; 24: 1079-87.

[31] Gamsjaeger S, Hofstetter B, Zwettler E, Recker R, Gasser JA, Erikssen EF, Klaushofer K, Paschalis EP. Effects of 3 years treatment with once-yearly zoledronic acid on the kinetics of bone matrix maturation in osteoporotic patients. Osteoporos. Int 2013; 24: 339-47.

[32] Dahl SG. Allain P, Marie PJ, Mauras Y, Boivin G, Ammamm P, Tsouderos Y, Delmas PD, Christiansen C. Incorporation and distribution of strontium in bone. Bone2001; 28: 446-53.

[33] Reginster JY, Kaufman JM, Goemaere S, Devogelaer JP, Benhamou CL, FElsenberg D, Diza_curiel M, Brandi ML, Badurski J, Wark J, Balogh A, Bruyére $O$, Roux C. Maintenance of antifracture efficacy over 10 years with strontium ranelate in postmenopausal osteoporosis. Osteoporos. Int 2012; 23: 1115-1122.

[34] Bunyaratavej N. The action of Strontium ranelate: myth or reality. J. Med. Assoc. Thai 2011; 94 Suppl 5: S35-37.

[35] Black DM, Reid IR, Cauley JA, Cpsman F, Leung PC, Lakatos P, Lippuner K, Cmmings SR, Hue TF, Mukhopadhyay A, Tan M, Aftring RP, Eastell R. The effect of 6 versus 9 years of zoledronic acid treatment in osteoporosis: a randomized second extension to the HORIZON-Pivotal Fracture Trial (PFT). J. Bone Miner. Res 2015; 30: 934-44. 